\section{Visión Electrónica Más que un estado sólido \\ https://doi.org/10.14483/issn.2248-4728}

UNIVERSIDAD DISTRITAL

FRANCISCO JOSÉ DE CALDAS

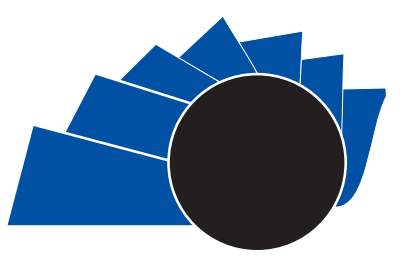

VISIÓN ELECTRONICA

Visión de caso

\title{
Generación de condensado con celdas de Peltier
}

\author{
Generation of condensate with Peltier cell \\ Yeison Dubiel Moreno Parra ${ }^{1}$, Henry Erik Pérez Valdeblanquez ${ }^{2}$, \\ Enrique Yamid Garzón González ${ }^{3}$
}

\section{INFORMACIÓN DEL ARTICULO}

Historia del articulo

Enviado: 10/11/2017

Recibido: 23/02/2018

Aceptado: 01/06/2018

\section{Palabras clave:}

Agua

Ambiente

Humedad

Temperatura

Keywords:

Water

Environment

Humidity

Temperature

\section{RESUMEN}

En este artículo se detalla la construcción de un dispositivo que emplea las celdas de peltier como forma de obtener agua del medio ambiente mediante la condensación. Para lograrlo se optó por utilizar la tarjeta de desarrollo Freescale FRDM- KL25Z, que permite regular la tensión que llega a la celda mediante una modulación por PWM. Adicionalmente el microcontrolador se encarga de la lectura de variables medio ambientales como la humedad relativa, la temperatura ambiente y la temperatura sobre la cara fría de una Celda de Peltier. Para así poder variar el PWM manteniendo la temperatura de la cara fría de la celda por debajo del punto de roció obteniendo un condensado y un posible goteo de agua.

\section{ABSTRACT:}

This article details the process of the construction of a device that uses peltier cells as a way to obtain water from the environment through condensation. To achieve this, we chose to use the Freescale FRDM-KL25Z development board, which allows regulating the voltage reaching the cell through PWM modulation. In addition, the microcontroller is responsible for reading environmental variables such as relative humidity, ambient temperature and temperature on the cold side of a Peltier cell. In order to be able to vary the PWM keeping the temperature of the cold side of the cell below the point of spray obtaining a condensate and a possible water drip.

\footnotetext{
1 Tecnólogo en Electrónica. Universidad Distrital Francisco José de Caldas, Colombia. Lugar de trabajo: Técnico en investigación y desarrollo en la empresa de telecomunicaciones Wodem. Correo electrónico: yeison9481@gmail.com. ORCID: https://orcid.org/0000-0002-6182-3209.

2Tecnólogo en Electrónica. Universidad Distrital Francisco José de Caldas, Colombia. Lugar de trabajo: Teleperformance, each interaction matters. Correo electrónico: henry.perez@teleperformance.com. ORCID: https://orcid.org/0000-0002-7028-6775.

SIngeniero en control electrónico e instrumentación, Universidad Distrital Francisco José de Caldas, Colombia. Especialista en Instrumentación Electrónica, Universidad Santo Tomás, Colombia. MSc. En Ingeniería Electrónica, Pontificia Universidad Javeriana, Colombia. Docente Universidad Distrital Francisco José de Caldas, Colombia. Correo electrónico: eygarzong@udistrital.edu.co. ORCID: https://orcid.org/0000-0001-9498-6349.

Citar este artículo como: Y. D. Moreno-Parra, H. E. Pérez-Valdeblanquez y E. Y. Garzón-González, "Generación de condensado con celdas de Peltier", Visión electrónica, algo más que un estado sólido, vol. 1, no. 1, Edición especial, enero-junio 2018. DOI revista: https://doi.org/10.14483/issn.224.8-4728. 


\section{Introducción}

El recurso vital para todos los seres humanos es el agua, pero lamentablemente este se ve en la actualidad amenazada por diversos factores por acción de la mano del mismo hombre, como la contaminación, el vertimiento de desechos altamente tóxicos a los recursos hídricos, desperdicio y la sobrepoblación entre muchos otros. En el caso colombiano no cambia mucho el panorama seguimos padeciendo de los mismos problemas, es por eso que en la actualidad se buscan nuevas maneras de obtención de recursos hídricos, un claro ejemplo son los dispositivos capaces de producir agua a partir de la condensación del aire.

Para esto se pensó en aprovechar los efectos principales de la termoelectricidad, como el efecto Peltier este explica el funcionamiento de la Celda de Peltier, la cual comprende de una placa cerámica de dos caras una que se eleva a altas temperaturas y la otra a muy bajas temperaturas, mediante la inducción de una corriente. Ya se han presentado diferentes proyectos en la actualidad sobre el uso de las Celdas de Peltier, en España han registrado una patente titulada, "SISTEMA Y DISPOSITIVO CONDENSADOR DE RECOGIDA DE AGUA DEL MEDIO AMBIENTE" [1].

Dispositivo que tiene como objetivo la recolección de agua mediante Celdas de Peltier con la particularidad de que esta riegue directamente las plantas de los cultivos aprovechando la energía del sol para el funcionamiento del sistema. Mediante sensores determina la temperatura ambiente para poder establecer la temperatura adecuada en las celdas, cumpliendo con el objetivo principal de recolección de agua. [1]. Cabe aclarar que el dispositivo anterior está diseñado solamente para trabajo agrícola mas no como fuente de agua en una comunidad.

Ahora teniendo un concepto claro de cómo se puede aprovechar el efecto Peltier surge la idea de construir en primera instancia un prototipo capaz de aprovechar la cara de la celda que se encuentra a bajas temperaturas, generando agua a partir de la condensación del aire que se encuentra en el ambiente, con el objetivo de diseñar un dispositivo que cumpla con tal fin, también se tuvieron en cuenta otros elementos como la temperatura ambiente, la humedad relativa y el punto de roció, variables que se lograron obtener mediante sensores electrónicos específicos, que son leídos y visualizados mediante una pantalla LCD y de la tarjeta de desarrollo, dispositivo que cuenta con un microcontrolador central y una serie de entradas o salidas.

Utilizando las variables anteriores a determinados valores se controlaron las celdas, mediante un sistema llamado PWM (Modulación por Ancho de Banda), que cuenta con un sistema de potencia permitiendo entregar los parámetros necesarios de voltaje y corriente a cada una de las celdas para así permitirles fijarse en la temperatura optima por debajo del punto de roció para el propósito establecido que es el del condensado del aire se cumpla. Este documento se enfoca en describir la realización del proyecto Generación de Condensado con Celdas de Peltier, se hará una descripción de cada etapa del dispositivo, se presentarán los resultados obtenidos, y finalmente las conclusiones. Este proyecto se ejecuta como primera etapa de un planteamiento del grupo de investigación DIGITI perteneciente a los programas de tecnología en electrónica, ingeniería en control e ingeniería en telecomunicaciones, de la Universidad Distrital Francisco José de Caldas, los cuales buscan el diseño de un dispositivo que sea capaz de generar agua, auto sostenible energéticamente, portable y eficiente.

\section{Desarrollo del proyecto}

Para entender la base de funcionamiento de este proyecto es necesario estudiar la termoelectricidad y cada uno de sus efectos en especial el efecto Peltier, el cual afirma que a la unión de dos metales diferentes sometidos a una corriente eléctrica aparece una diferencia de temperaturas. La dirección de la transferencia de calor es controlada por la polaridad de la corriente. Al invertir la polaridad se cambia la dirección de la transferencia y, como consecuencia, la unión donde se desprendía calor lo absorberá y donde se absorbía lo desprenderá, al hacer pasar la corriente eléctrica por un circuito compuesto de materiales distintos y con sus uniones a la misma temperatura se obtiene el efecto inverso absorbiendo calor por una de las uniones y desprendiéndose de la otra. El efecto anterior dicta el principio fundamental de la Celda Peltier, base primordial para el desarrollo óptimo y buen funcionamiento del dispositivo diseñado.

Para que las celdas de Peltier trabajen en las condiciones más adecuadas se deben tener en cuenta las variables de temperatura ambiente y de la humedad relativa, en la figura 1 que se presenta a continuación se muestra las diferentes condiciones medio ambientales promedio de las principales zonas geográficas de Colombia [2], para saber mediante el punto de roció, en que regiones podría funcionar de una manera más óptima. 


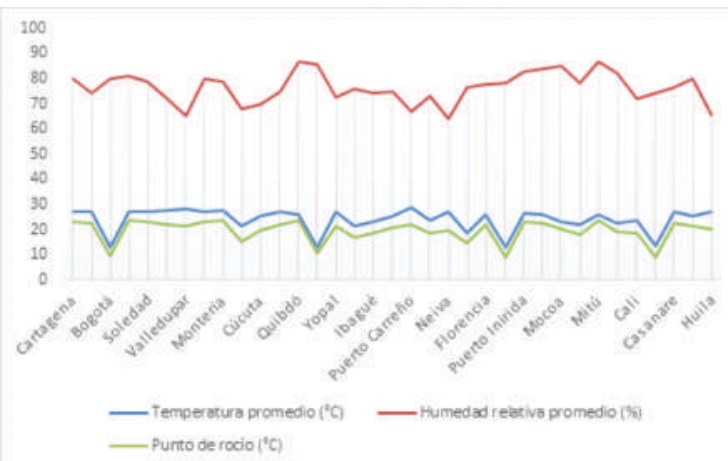

Figura 1. Condiciones medioambientales por regiones [2].

En la figura anterior se puede observar que se ha realizado un cálculo del punto de rocío promedio para cada región, por lo que se puede estimar que en zonas donde la temperatura ambiente y el punto de rocío son más cercanos se requerirá de una menor potencia para obtener un condensado.

Por otra parte, es claro que existen lugares en el país donde la humedad relativa promedio supera el $80 \%$, condición ideal para que el dispositivo recolecte cantidades moderadamente buenas de agua, zonas donde se presentan bajos niveles de escorrentía como la península de la guajira también tienen humedades relativamente buenas para que el dispositivo sea capaz de recolectar cantidades menores de agua. Sin embargo, esto no descarta la posibilidad de que el dispositivo no sea funcional en todas las regiones del país ya que para todos los valores de humedad relativa se podría obtener un condensado del aire en cualquier región del país.

Para explicar de mejor manera el dispositivo este se encuentra dividido en varias etapas como se muestra en la figura 2, las cuales son; unidad de procesamiento, sensores, potencia, fuente de energía, visualización y Celdas de Peltier.

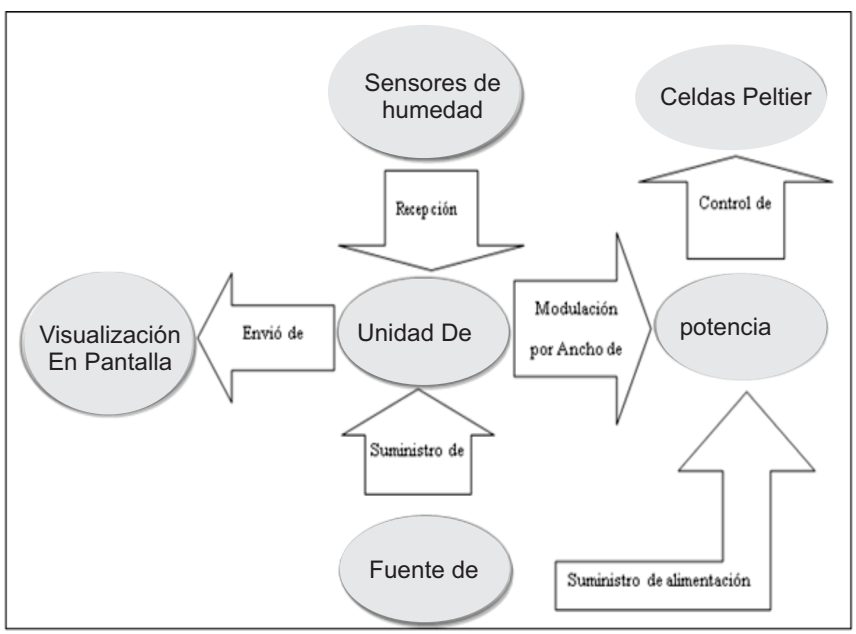

Figura 2. Diagrama general del Dispositivo. Fuente: elaboración propia.
Cada etapa será descrita a continuación, demostrando los elementos utilizados las características de cada uno y de los circuitos empleados para tal fin, en el esquema de la figura 3 se encuentran las respectivas conexiones entre cada etapa del dispositivo.

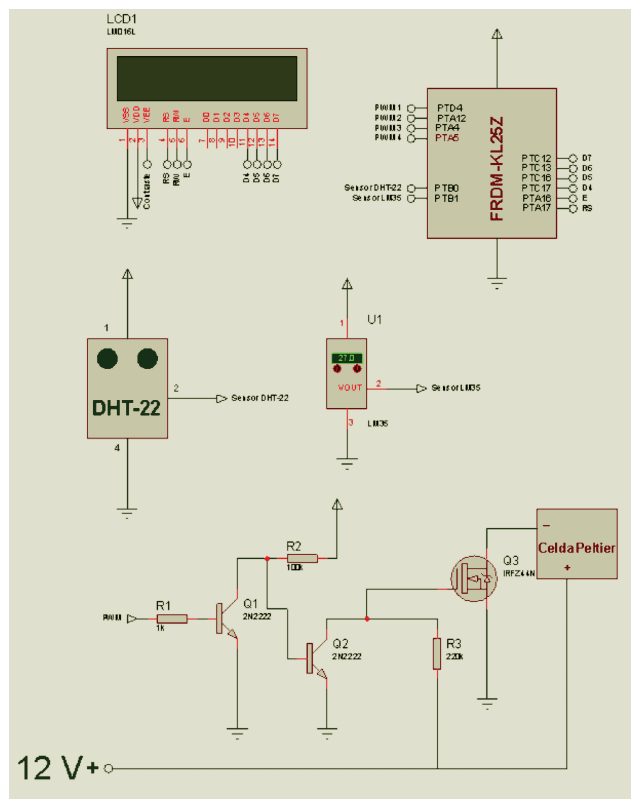

Figura 3. Esquema de conexiones del Dispositivo. Fuente: elaboración propia.

\subsection{Sensores de Humedad y Temperatura}

En el dispositivo se utilizaron dos sensores para controlar las variables físicas que permiten su correcto funcionamiento, el primer sensor es el LM35, utilizado para obtener la temperatura sobre la cara a bajas temperaturas sobre las celdas. Se optó por este sensor por ser fácil de usar, económico y preciso. En la figura 4 se encuentra la configuración sugerida por el fabricante para detectar temperaturas desde los $2{ }^{\circ} \mathrm{C}$ hasta los 150 ${ }^{\circ} \mathrm{C}$. El comportamiento de este es lineal, esta calibrado en grados Celsius y cada $10 \mathrm{mv}$ corresponden a $1{ }^{\circ} \mathrm{C}$. Una de sus mayores ventajas es que no se requiere de un circuito adicional para que este funcione como sucede en un termistor, un RTC, o un PTC.

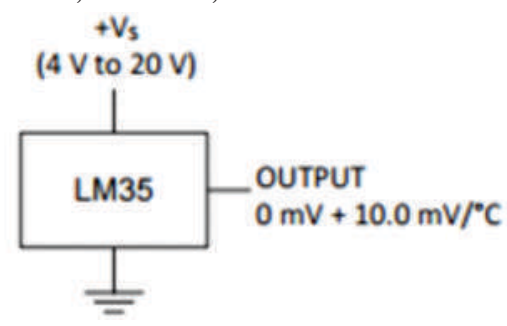

Figura 4. Configuración sugerida para sensor LM35 [3]. 
El segundo sensor que se utiliza es el DHT22, ofrece la posibilidad de leer las dos variables temperatura y humedad al tiempo, funciona en un rango de temperatura desde $-40{ }^{\circ} \mathrm{C}$ hasta $80{ }^{\circ} \mathrm{C}$. [4] Lee condiciones de humedad desde el $0 \%$ hasta el $99.9 \%$, maneja un tiempo de respuesta de 2 segundos, destacando su precisión por la facilidad de leer estas dos variables medio ambientales con un mismo dispositivo sin necesidad de conectar ningún otro componente lo hace el sensor ideal para calcular el punto de roció.

Adicionalmente se comprobó si los datos que se obtienen de ambos sensores son correctos para esto se utilizó el instrumento certificado AMPROBE TH-1 Relative Humidity / Temperature Probe Style Meter, que maneja una precisión de $+/-3 \%$ en humedad relativa $\mathrm{y}+/-0.6{ }^{\circ} \mathrm{C}$ en temperatura. [5]

Se tomaron 20 pruebas en total bajo diferentes condiciones medioambientales, en la tabla 1 se aprecia los datos obtenidos, el error absoluto y el error relativo. Para el error absoluto se restó el valor de temperatura obtenido por el DHT-22 y el LM35 al valor obtenido por el instrumento certificado, el error relativo se obtuvo multiplicando el absoluto por 100 y se dividió entre los datos del instrumento certificado.

\begin{tabular}{|c|c|c|c|c|c|c|c|}
\hline \multirow{3}{*}{ Prueba 1} & \multicolumn{3}{|c|}{ Temperatura $\left({ }^{\circ} \mathrm{C}\right)$} & \multirow{2}{*}{\multicolumn{2}{|c|}{$\begin{array}{c}\text { Error relativo } \\
\text { LM35 (\%)DHT22 }\end{array}$}} & \multirow{2}{*}{\multicolumn{2}{|c|}{$\begin{array}{l}\text { Error Absoluto } \\
\text { LM35 ('CDHT22 }\end{array}$}} \\
\hline & \multirow{2}{*}{$\begin{array}{c}\text { TH1 } \\
23,6\end{array}$} & \multirow{2}{*}{$\begin{array}{c}\text { LM35 } \\
24,08\end{array}$} & \multirow{2}{*}{\begin{tabular}{|r|} 
DHT22 \\
24,8
\end{tabular}} & & & & \\
\hline & & & & 2,03 & 5,08 & $-0,48$ & $-1,2$ \\
\hline Prueba 2 & 22,0 & 23,03 & 22,6 & 4,68 & 2,73 & $-1,03$ & $-0,6$ \\
\hline Prueba 3 & 25,3 & 23,88 & 22,9 & 5,61 & 9,49 & 1,42 & 2,4 \\
\hline Prueba 4 & 23,7 & 23,35 & 24,6 & 1,48 & 3,80 & 0,35 & $-0,9$ \\
\hline Prueba 5 & 26,0 & 24,30 & 24,6 & 6,54 & 5,38 & 1,7 & 1,4 \\
\hline Prueba 6 & 24,2 & 23,99 & 24,8 & 0,87 & 2,48 & 0,21 & $-0,6$ \\
\hline Prueba 7 & 24,7 & 24,11 & 24,9 & 2,39 & 0,81 & 0,59 & $-0,2$ \\
\hline Prueba 8 & 24,1 & 24,04 & 24,7 & 0,25 & 2,49 & 0,06 & $-0,6$ \\
\hline Prueba 9 & 25,6 & 23,65 & 23,9 & 7,62 & 6,64 & 1,95 & 1,7 \\
\hline Prueba 10 & 27,0 & 24,75 & 27,8 & 8,33 & 2,96 & 2,25 & $-0,8$ \\
\hline Prueba 11 & 27,8 & 24,84 & 27,8 & 10,65 & 0,00 & 2,96 & 0 \\
\hline Prueba 12 & 23,5 & 24,34 & 27,9 & 3,57 & 18,72 & $-0,84$ & $-4,4$ \\
\hline Prueba 13 & 25,8 & 24,34 & 25,3 & 5,66 & 1,94 & 1,46 & 0,5 \\
\hline Prueba 14 & 25,3 & 24,96 & 27,6 & 1,34 & 9,09 & 0,34 & $-2,3$ \\
\hline Prueba 15 & 27,9 & 25,37 & 27,5 & 9,07 & 1,43 & 2,53 & 0,4 \\
\hline Prueba 16 & 25,4 & 24,41 & 25,7 & 3,90 & 1,18 & 0,99 & $-0,3$ \\
\hline Prueba 17 & 24,6 & 25,05 & 28,0 & 1,83 & 13,82 & $-0,45$ & $-3,4$ \\
\hline Prueba 18 & 26,2 & 24,45 & 26,0 & 6,68 & 0,76 & 1,75 & 0,2 \\
\hline Prueba 19 & 23,5 & 24,14 & 27,8 & 2,72 & 18,30 & $-0,64$ & $-4,3$ \\
\hline Prueba 20 & 23,4 & 24,48 & 27,6 & 4,62 & 17,95 & $-1,08$ & $-4,2$ \\
\hline Promedio & 24,98 & 24,28 & 25,84 & 4,49 & 6,25 & 0,70 & $-0,86$ \\
\hline
\end{tabular}

Tabla 1. Pruebas de temperatura y errores. Fuente: elaboración propia.

Durante la prueba anterior se logró obtener la humedad relativa mediante el instrumento AMPROBE TH-1 para ser comparada con la obtenida por el sensor DHT22 y del mismo modo se encontró los errores absolutos y relativo, datos que se encuentran especificados en la tabla 2.

\begin{tabular}{|c|c|c|c|c|}
\hline & \multicolumn{2}{|c|}{ Humedad (\%) } & Error relativo & Error absoluto \\
\hline & TH1 & DHT22 & (\%) DHT & (\%) DHT \\
\hline Prueba 1 & 22,8 & 21,1 & 7,46 & 1,7 \\
\hline Prueba 2 & 23,7 & 21,3 & 10,13 & 2,4 \\
\hline Prueba 3 & 20,8 & 20,8 & 0,00 & 0 \\
\hline Prueba 4 & 20,9 & 19,4 & 7,18 & 1,5 \\
\hline Prueba 5 & 16,8 & 20,7 & 23,21 & $-3,9$ \\
\hline Prueba 6 & 19,7 & 19,8 & 0,51 & $-0,1$ \\
\hline Prueba 7 & 16,2 & 18,6 & 14,81 & $-2,4$ \\
\hline Prueba 8 & 21,0 & 20,3 & 3,33 & 0,7 \\
\hline Prueba 9 & 16,7 & 18,8 & 12,57 & $-2,1$ \\
\hline Prueba 10 & 15,6 & 17,2 & 10,26 & $-1,6$ \\
\hline Prueba 11 & 18,6 & 17,7 & 4,84 & 0,9 \\
\hline Prueba 12 & 17,5 & 13,8 & 21,14 & 3,7 \\
\hline Prueba 13 & 16,5 & 18,1 & 9,70 & $-1,6$ \\
\hline Prueba 14 & 16,6 & 18,6 & 12,05 & $-2,0$ \\
\hline Prueba 15 & 16,2 & 18,4 & 13,58 & $-2,2$ \\
\hline Prueba 16 & 16,0 & 16,9 & 5,62 & $-0,9$ \\
\hline Prueba 17 & 17,3 & 15,8 & 8,67 & 1,5 \\
\hline Prueba 18 & 16,1 & 17,2 & 6,83 & $-1,1$ \\
\hline Prueba 19 & 17,8 & 13,9 & 21,91 & 3,9 \\
\hline Prueba 20 & 19,0 & 16,2 & 14,74 & 2,8 \\
\hline Promedio & 18,29 & 18,23 & 10,43 & 0,06 \\
\hline
\end{tabular}

Tabla 2. Pruebas de humedad relativa y errores.

Fuente: elaboración propia.

A partir de las pruebas anteriores se pudo determinar que las medidas de los sensores utilizados en el dispositivo son confiables ya que los promedios de los errores no son tan elevados.

\subsection{Unidad de Procesamiento}

Se utilizó la tarjeta de desarrollo FRDM-KL25Z ya que es un dispositivo que ofrece facilidades con su entorno de programación y es diseñada como una plataforma de bajo costo, su programación se hace por un compilador en línea que tiene la ventaja de poder trabajar en cualquier dispositivo que cuente con una conexión a internet.

En la figura 5 se puede apreciar los pines utilizados en total 11, uno análogo y el resto digitales.

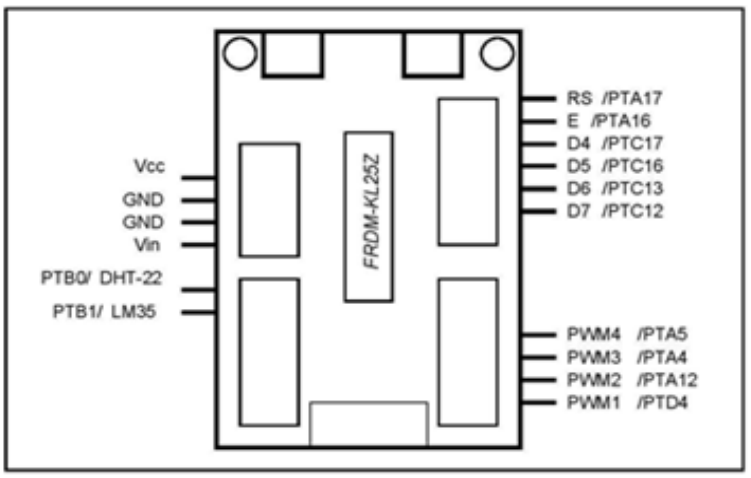

Figura 5. GPIO utilizados de la FRDM-KL25Z. Fuente: elaboración propia.

El código fue escrito en un lenguaje que se basa en $\mathrm{C}++$, junto con librerías que permiten leer fácilmente el sensor DHT22 y el sensor $\operatorname{lm} 35$, al conectar el dispositivo el código obtiene una temperatura y una humedad relativa del sensor DHT22, con lo cual se 
dispositivo el código obtiene una temperatura y una humedad relativa del sensor DHT22, con lo cual se calcula el punto de roció, una vez se tiene la temperatura del punto de roció se sabe que la temperatura de la celda se debe colocar inferior a eso, pero superior a $\operatorname{los} 0^{\circ}$ Celsius, por lo que se le envía una señal de PWM de $500 \mathrm{~Hz}$ a la celda, con un ciclo útil que comienza a aumentar y se detiene cuando se alcanza una temperatura inferior a dicho punto. Adicionalmente los datos relevantes como la temperatura de los sensores, la humedad, el punto de roció y el porcentaje del PWM son trasmitidos constantemente por USB como puerto serial a una velocidad de 9600 baudios, de manera que se puede observar dichos valores desde un computador con un simulador de puerto serie como el hyperterminal. Así mismo las variables de temperatura ambiente y humedad relativa son impresas constantemente en una pantalla LCD 16X2 que se encuentra en el dispositivo con fácil visibilidad para el usuario. El diagrama de flujo del código asociado a esta explicación se observa en la Figura 6.

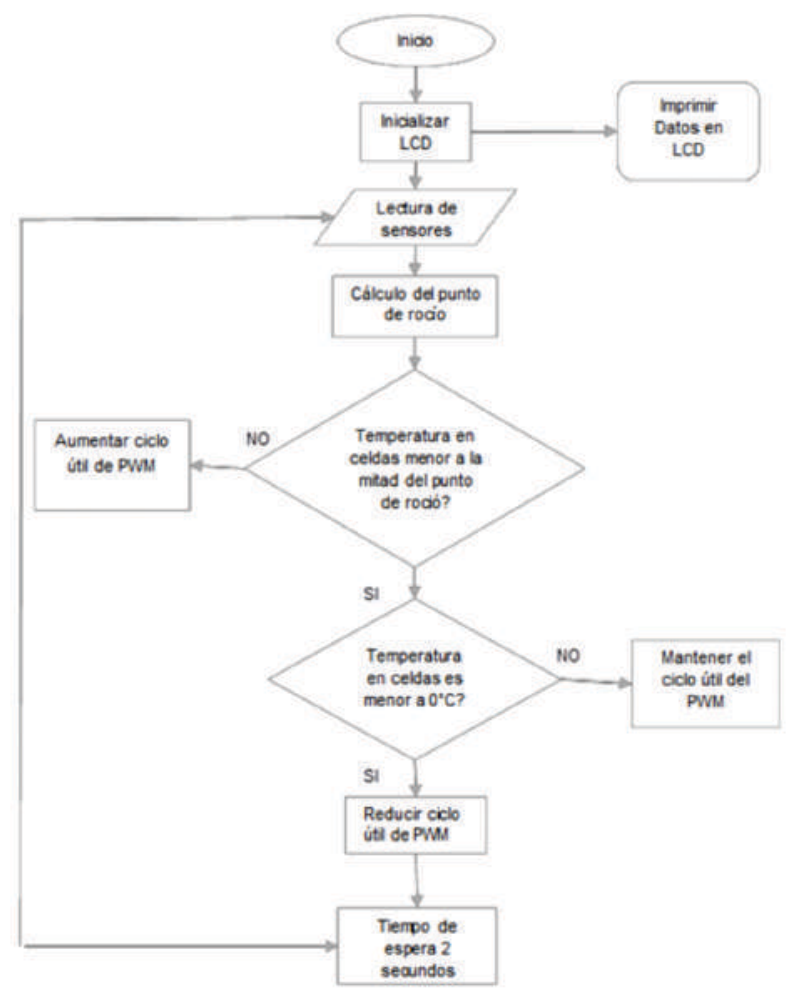

Figura 6. Diagrama de flujo del sistema general. Fuente: Elaboración propia.

\subsection{Celdas Peltier}

La Celda de efecto Peltier es un dispositivo termoeléctrico semiconductor el cual al hacer circular una corriente por él, aparece una diferencia de temperatura entre sus caras, es decir, una de sus caras se calienta y la otra se enfría, como puede verse en a figura 7 la celda está formada por dos conexiones por donde llega la corriente continua a la celda, dos superficies fabricadas con material cerámico aislante, las cuales se pondrán más frías o más calientes en función de la polaridad de la corriente y de la cantidad de amperios que se hagan circular por la celda. Por último, la célula está fabricada en su parte interior con un material conductor, este material está compuesto por dos metales diferentes o semiconductores tipo $\mathrm{P}$ y tipo $\mathrm{N}$ que están conectados entre sí. $\lceil 6\rceil$

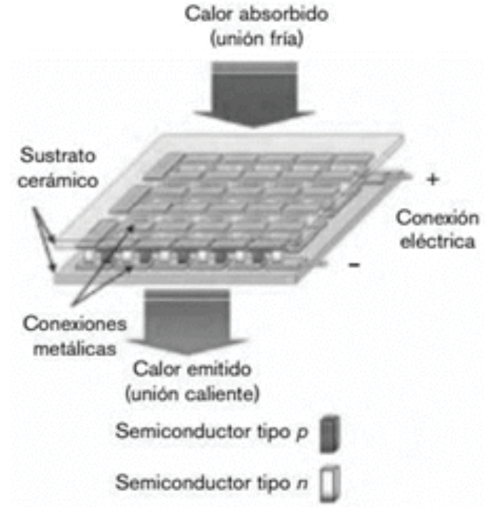

Figura 7. Esquema de las partes de la célula de efecto Peltier [6].

Para este dispositivo se utilizó la celda TEC1-12706 siendo la más común en el mercado, fabricadas en cerámica y semiconductores de tipo $\mathrm{P}$ y tipo $\mathrm{N}$, principalmente son celdas de 12 voltios y 6 amperios como máximo, manejan una temperatura máxima de hasta $65{ }^{\circ} \mathrm{C}$ y sus dimensiones son de $4 \times 4 \times 0.4 \mathrm{~cm}$, llegando a pesar un máximo de $22 \mathrm{~g}$, como se observa en la figura 8 .

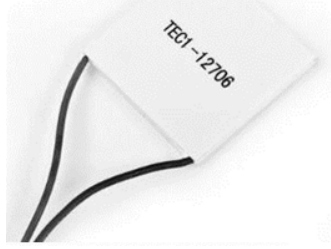

Figura 8. Modelo de celda Peltier utilizada [7].

Para que el dispositivo sea más eficiente a la hora de condensar agua, se incrementó el área de condensación mediante un arreglo de cuatro Celdas Peltier TEC112706, estas se encuentran fijadas a una placa de aluminio o disipador y se basan en la ley cero de la 
termodinámica transfiriendo el calor de la parte caliente que se desea disipar de una mayor área por la cara que se eleva a altas temperaturas con el fin de evitar daño alguno sobre la celda. Sin embargo, también se fijó en la parte posterior un ventilador que funciona como una evacuación de calor en los disipadores hacia el exterior permitiendo un sistema de mayor refrigerado. El ventilador trabaja a un voltaje determinado de $12 \mathrm{~V}$ y a una corriente máxima de $240 \mathrm{~mA}$.

\subsection{Potencia}

Para lograr que las celdas de Peltier consigan una temperatura muy baja, se necesita una corriente elevada para esto se utilizó el circuito que se observa en la figura 9, es un circuito de control mediante un PWM (Modulación por Ancho de Pulsos), técnica que se basa en la modificación del ciclo útil para cambiar los niveles de tensión que se envía a una carga. [7] Se conecta y desconecta 500 veces en un segundo ósea maneja una frecuencia de $500 \mathrm{~Hz}$, controlando la corriente suministrada desde la fuente. Consta de dos transistores 2n2222, transistores bipolares NPN de baja potencia que sirven como etapa preamplificadora de la señal y un MOS-FET de alta potencia IRFZ44N, este maneja corrientes de hasta $50 \mathrm{~A}$ siendo este uno de los más ideales sin temor a sufrir sobrecalentamientos, con una resistencia tan baja que puede llegar hasta los 0.017 ohms. Permitiendo un trabajo excelente ya que al máximo de sus posibilidades no desarrollo una potencia mayor a los $45 \mathrm{~W}$, para trabajar el PWM con el MOSFET se deben manejar los tiempos y las formas de los flancos de subida y bajada de la señal.

Al enviar la señal PWM desde el microcontrolador llega a la resistencia de $1 \mathrm{k}$ esta atenúa un poco el voltaje entra a la base de Q1, mientras que el colector maneja una resistencia de $100 \mathrm{k}$ conectada directamente a VCC, esto permite controlar la corriente que pasa hacia $\mathrm{Q}^{2}$ por la base conectando al colector la resistencia de 220 $K$, funcionando como etapa preamplificadora hacia el MOS-FET IRFZ44N por Gate, este último funciona mediante corte y saturación entre Source y Drain, polarizando una de las Celdas de Peltier TEC1-12706. El circuito se encuentra en una PCB (Placa de circuito impreso), adicionalmente se maneja disipadores de aluminio en el MOS-FET IRFZ44N para evitar sobrecalentamientos y generar daños sobre el mismo.

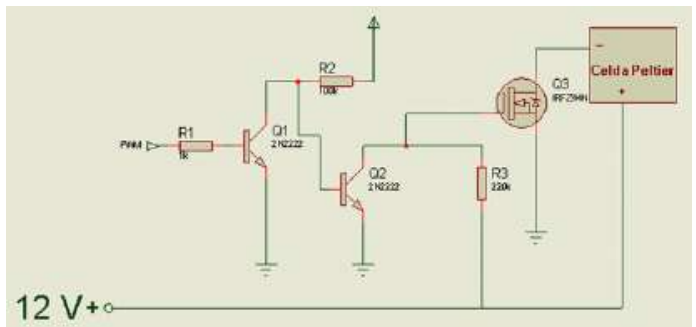

Figura 9. Circuito de Potencia. Fuente: elaboración propia..

\section{Pruebas y resultados}

El dispositivo final para este proyecto se considera como la primera etapa del planteamiento propuesto por el grupo de investigación DIGITI, expuesto al principio del documento. Se logra construir un dispositivo de base cuadrada con las siguientes medidas; $30 \mathrm{~cm}$ de largo con $25 \mathrm{~cm}$ de ancho y una altura de $15 \mathrm{~cm}$. Además, se implementó un sistema de recolección de $\mathrm{H}_{2} \mathrm{O}$; cilindro con diámetro de $11 \mathrm{~cm} \mathrm{y}$ una altura de $25 \mathrm{~cm}$. El cual cuenta con una manguera para evacuar el agua almacenada y ver su nivel. En la figura 10 se puede apreciar el dispositivo final en detalle.

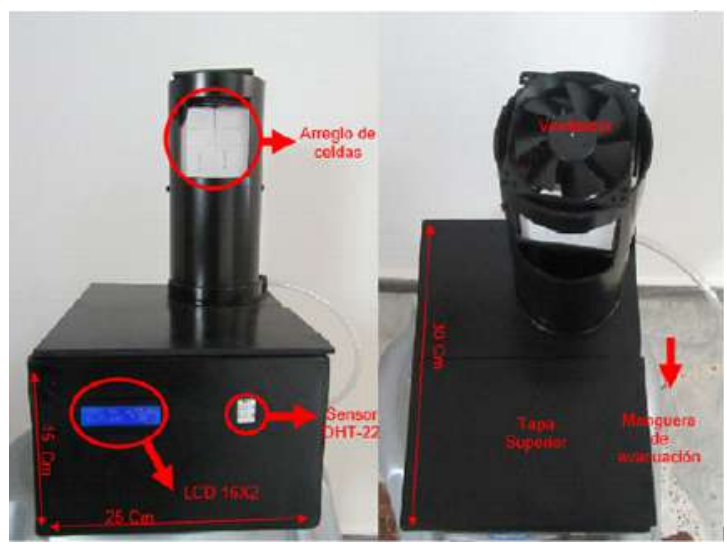

Figura 10. Vista frontal y superior del dispositivo. Fuente: elaboración propia.

En la figura anterior se puede apreciar las diferentes partes del dispositivo, el cual es una caja y en el interior contiene; la fuente de poder, la tarjeta de desarrollo, el circuito impreso de potencia y el cableado necesario para el buen funcionamiento del dispositivo. En la parte externa se encuentra el sistema de recolección de $\mathrm{H} 2 \mathrm{O}$, dentro del cual se tienen el arreglo de celdas y a su vez esta acoplado el sensor LM35, el disipador y los dos ventiladores. En la parte frontal se encuentra la pantalla LCD 16X2 y el sensor DHT-22, finalmente en 
la parte superior se cuenta con una tapa con bisagras que permite fácilmente la visualización y su manipulación. Es capaz de medir la temperatura ambiente, la humedad relativa y calcular el punto de roció, estas variables se van a poder visualizar en una pantalla LCD de 16x2, con una tasa de trasferencia de datos de cada 2 segundos. La temperatura obtenida por el sensor LM35 sobre la cara fría de la celdas, es comparada con el punto de roció, si este último es menor a la temperatura obtenida por el sensor LM35, el sistema debe subir el PWM para que las celdas funcionen a una corriente mayor y descienda su temperatura hasta que esta logre ser igual o menor entre el punto de roció y la obtenida del sensor LM35, esto con tal de garantizar que las celdas se encuentren a la temperatura ideal para efectuar un condensado como se muestra en la figura 11. Mientras por el otro lado de las celdas la cara caliente se eleva a temperaturas cercanas a los $100{ }^{\circ} \mathrm{C}$, para lograr descender esta temperatura se inicia un ventilador capaz de disipar el calor en las placas de aluminio donde se encuentran las celdas evitando así un sobrecalentamiento y daño del sistema.

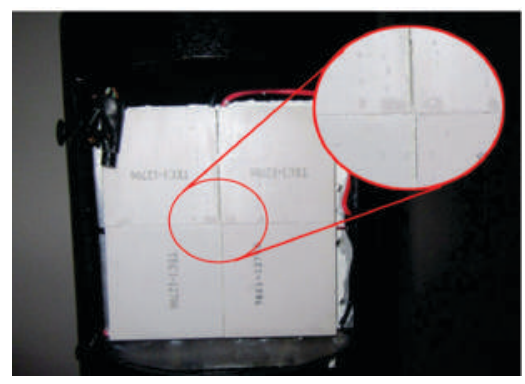

Figura 11. Condensado sobre la cara fría de las celdas. Fuente: elaboración propia.

Durante las primeras pruebas se recolectaron los datos de voltaje, corriente y temperatura sobre la cara fría de una sola celda que se aprecian en la tabla 3. Con la finalidad de conocer voltaje y corriente máximo con la que se produce la temperatura de congelamiento del agua la cual es $\mathrm{O}^{\circ} \mathrm{C}$.

\begin{tabular}{|r|r|r|}
\hline Voltaje (V) & Corriente $(\mathbf{m A})$ & Temperatura $\left({ }^{\circ} \mathbf{C}\right)$ \\
\hline 0,5 & 130 & 21 \\
\hline 1 & 280 & 18 \\
\hline 1,5 & 400 & 16 \\
\hline 2 & 560 & 14 \\
\hline 2,5 & 680 & 10 \\
\hline 3 & 850 & 7 \\
\hline 3,5 & 990 & 5 \\
\hline 4 & 1140 & 4 \\
\hline 4,5 & 1310 & 2 \\
\hline 5 & 1440 & 1 \\
\hline 5,5 & 1605 & 1 \\
\hline 6 & 1740 & 0 \\
\hline
\end{tabular}

Tabla 3. Voltaje, corriente y temperatura en una Celda Peltier. Fuente: elaboración propia.
A partir de la tabla anterior se puede establecer una relación de tipo lineal entre el voltaje y la corriente como se muestra en la figura 12.

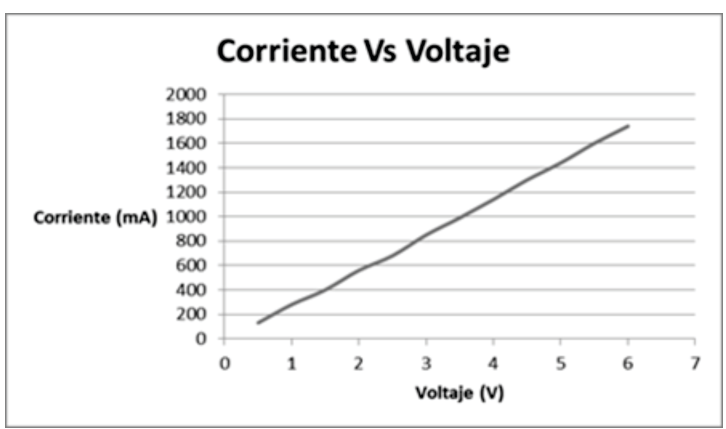

Figura 12. Grafica de la relación corriente y voltaje en una Celda Peltier. Fuente: elaboración propia.

Se hizo una prueba específica para determinar la cantidad de agua que se podría recolectar con el dispositivo final de 4 celdas, bajo los parámetros que se encuentran en la tabla 4.

\begin{tabular}{|c|c|c|c|}
\hline Duración & $\begin{array}{c}\text { Temperatura } \\
\text { ambiente }\end{array}$ & $\begin{array}{c}\text { Humedad } \\
\text { relativa }\end{array}$ & $\begin{array}{c}\text { Punto de } \\
\text { rocío }\end{array}$ \\
\hline 4 horas & $22^{\circ} \mathrm{C}$ & $34 \%$ & 5.37 \\
\hline
\end{tabular}

Tabla 4. Parámetros de la prueba. Fuente: elaboración propia.

Pasadas las 4 horas se observó que la cantidad de agua recogida era aproximada a los $20 \mathrm{ml}$, se midió la temperatura de las celdas por la cara fría y estaba cerca de $\operatorname{los} 5{ }^{\circ} \mathrm{C}$.

Posteriormente se realizaron pruebas para determinar la eficiencia entre la potencia consumida y el agua recolectada bajo los mismos parámetros establecidos de la tabla 4, con la diferencia que la duración solo fue de una hora para cada valor de potencia. En la figura 13 se muestra los valores de la potencia instantánea consumida con relación al agua recolectada.

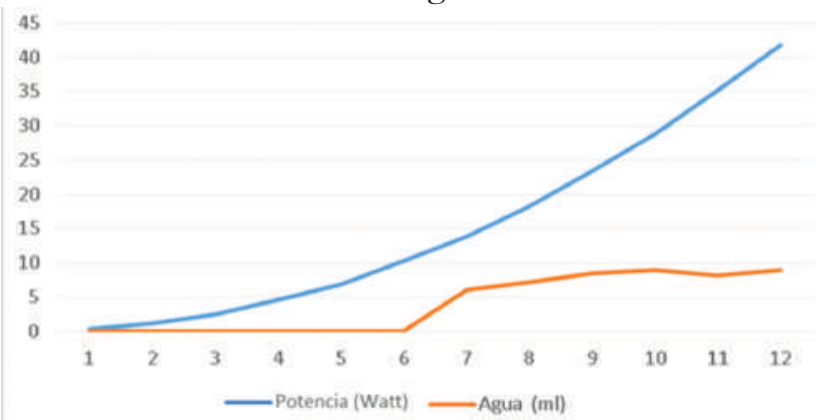

Figura 13. Potencia instantánea y Agua recolectada. Fuente: elaboración propia. 
A partir de la gráfica anterior podemos observar que el dispositivo comienza a condensar cuando la potencia es suficiente para que la temperatura en las caras de las celdas sea inferior al punto de rocío, para estas pruebas la potencia inicial de condensación es de $13.86 \mathrm{~W}$, proporcionando aproximadamente $6 \mathrm{ml}$ de agua en una hora.

La siguiente prueba se desarrolló en horas nocturnas, bajo las condiciones que se encuentran en la tabla 5 , para conocer el comportamiento del dispositivo bajo otras condiciones medioambientales.

\begin{tabular}{|c|c|c|c|}
\hline Duración & $\begin{array}{c}\text { Temperatura } \\
\text { ambiente }\end{array}$ & $\begin{array}{c}\text { Humedad } \\
\text { relativa }\end{array}$ & $\begin{array}{c}\text { Punto de } \\
\text { rocío }\end{array}$ \\
\hline 2 horas & $15^{\circ} \mathrm{C}$ & $42 \%$ & 2.1 \\
\hline
\end{tabular}

Tabla 5. Parámetros de la prueba. Fuente: elaboración propia.

En esta prueba se aumentó el voltaje $0.5 \mathrm{~V}$ cada dos horas para aumentar la potencia de la misma forma que la prueba anterior, para conocer el agua recolectada en los diferentes intervalos como se observa en la figura 14 .

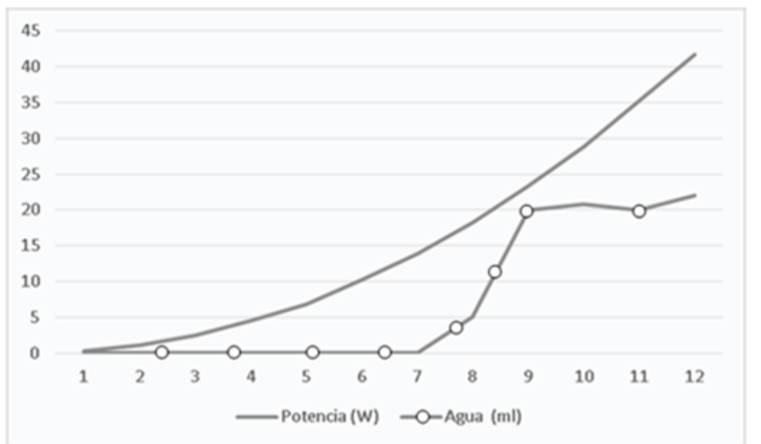

Figura 14. Potencia instantánea y Agua recolectada. Fuente: elaboración propia.

Comprobando así que en horas de la noche la producción de agua es mayor, esto se debe a que la humedad relativa también lo es. Por otra parte, se observa el mismo comportamiento, el dispositivo comienza a condensar desde que la temperatura de la cara de la celda es inferior al punto de rocío (para este caso inferior a 2.1) y comienza a trabajar a partir de 23.4 W.

\section{Conclusiones}

Teniendo en cuenta las pruebas de medición de temperatura ambiente y humedad relativa con el instrumento certificado AMPROBE TH-1, se puede determinar que los sensores DHT22 y LM35 utilizados en el dispositivo, funcionan de una manera correcta ya que el promedio de error relativo es de $6.25 \%$ y $4.49 \%$ respectivamente, tan solo para temperaturas mientras que en humedad relativa maneja un error de $10.43 \%$.

Debido a que la potencia en las celdas de Peltier crece de forma exponencial conforme se aplica más voltaje se generan altas corrientes. Por celda se puede llegar a alcanzar 2.5 A como máximo, por lo anterior se hizo necesario la utilización de un circuito donde funcione un Mosfet de potencia capaz de aguantar hasta $50 \mathrm{~A}$, características que nos permiten evitar que sufra de calentamientos excesivos y funcione durante largos periodos sin problema.

Finalmente, el aumento de la cantidad de celdas TEC112706 de una a cuatro, incide directamente en la producción de agua en grandes cantidades, de $5 \mathrm{ml}$ a 20 $\mathrm{ml}$ según estudios previos. El uso de este dispositivo es ideal en lugares donde la humedad relativa es cercana o superior al $70 \%$ y su punto de roció se encuentre entre los $18{ }^{\circ} \mathrm{C}$ y $25^{\circ} \mathrm{C}$, llegando a obtener mayores niveles de condensación y de goteo constante, que permita recoger mayores cantidades de agua llegando a solventar en un porcentaje bajo la escasez de este recurso.

\section{Referencias}

[1] M. A. Muñoz García, A. Soler Esteban y S. Van Olmen, "Sistema y dispositivo condensador de recogida de agua del medio ambiente", Universidad Politécnica de Madrid, España, 2012.

[2] IDEAM, "Características climatológicas de ciudades principales y municipios turísticos", 2013. [En línea]. Disponible en: http://www.ideam.gov.co/documents/21021 /21789/1Siti\%20os+turisticos2.pdf/cd4106e 9-d608-4c29-91cc-16bee9151ddd.

[3] Texas Instruments, "LM $35 \pm 0.5^{\circ} \mathrm{C}$ Temperature Sensor with Analog Output and 30V Capability", 2016. [En línea]. Disponible en: http://www.ti.com/product/LM35.

[4] T. Liu, "Digital-output relative humidity \& temperatura sensor/module DHT22 (DHT22 also named as AM2302)", [En línea]. A v a i l a b l e.

https://www.sparkfun.com/datasheets/Sens ors/Temperature/DHT22.pdf.

[5] AMPROBE, "TH-1 Relative Humidity / Temperature Probe Style Meter", 2012. [En 
lín e a J. D i s p o n i b l e e n : http://content.amprobe.com/manualsA/TH1_Manual_all.pdf.

[6] Hebei I. T. (Shanghai) Co., Ltd., “Thermoelectric Cooler TEC1-12706". [En lín e a ]. D i s p o n i b l e e n : http://peltiermodules.com/peltier.datasheet/ TEC1-12706.pdf.

[7] HB Corporation, "TEC1-12706 Datasheet", [En lín e a ]. D i s p o n i ble e n : http://pdf1.alldatasheet.com/datasheetpdf/view/227422/ETC2/TEC1-12706.html. 